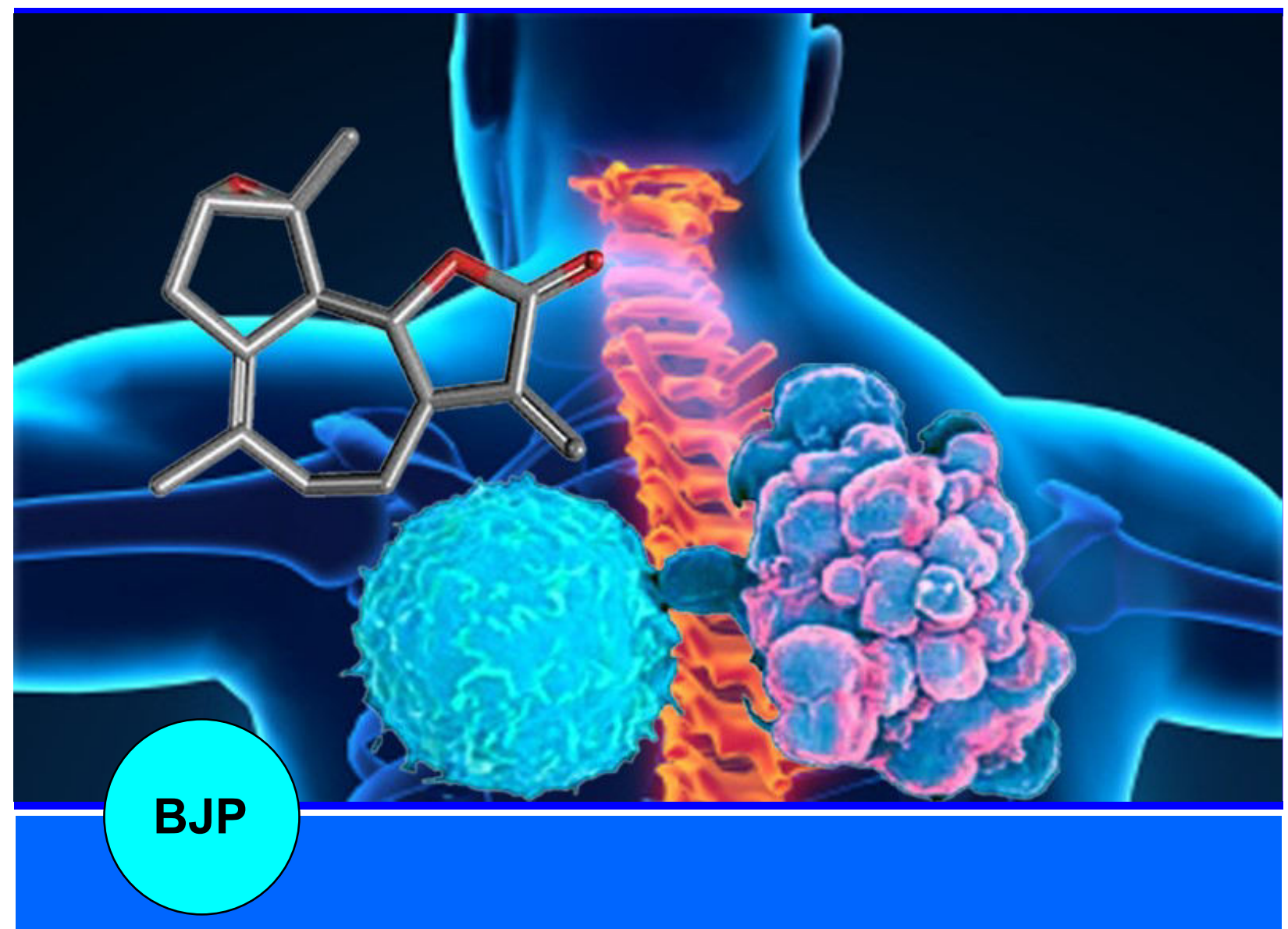

Bangladesh Journal of Pharmacology

Research Article

Ludartin exhibits therapeutic effect on spinal cord injury through inhibition of apoptosis and inflammation 


\title{
Ludartin exhibits therapeutic effect on spinal cord injury through inhibition of apoptosis and inflammation
}

\author{
Weiyuan $\mathrm{Xu}^{1}$, Shenglong Miao² and Yuchen Feng3 \\ ${ }^{1}$ Department of Orthopedics, Lanzhou University Second Hospital, Lanzhou 730030, China; ${ }^{2}$ Department of \\ Orthopedics, Wuwei People's Hospital, Wuwei 733000, China; ${ }^{3}$ Second Clinical Medical College, Lanzhou \\ University, Lanzhou 730030, China.
}

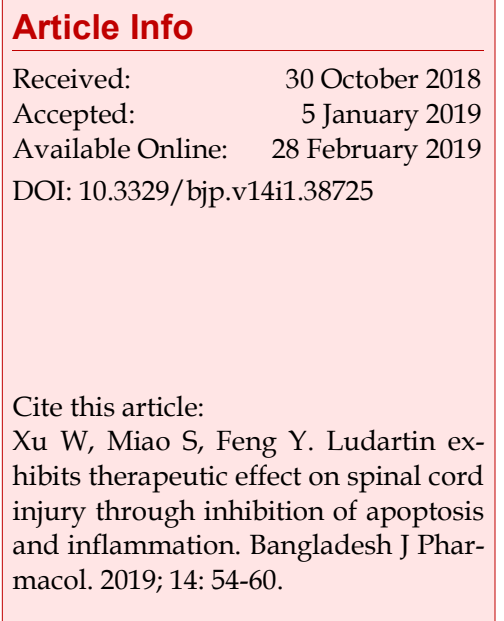

\begin{abstract}
The presents study investigates the effect of ludartin on spinal cord injury in rat model. Ludartin treatment decreased the expression of myeloperoxidase and malondialdehyde in spinal cord tissues. The expression of glutathione and superoxide dismutase were enhanced. It also exhibited inhibitory effect on reduction of NeuN-positive cells. The proportion of TUNEL positive cells was also regulated. In addition, ludartin treatment prevented the onset of apoptosis which was evident by decrease in caspase- 3 and Bax level and increase of Bcl-2 level. Up-regulation of tumor necrosis factor- $\mathrm{a}$, interleukin- $1 \beta$ and I interleukin- 6 by spinal cord injury was suppressed by ludartin treatment. There was improvement in locomotion of rats by treatment with ludartin. Ludartin treatment of spinal cord injury rats improves locomotion by inhibiting inflammatory cytokine expression and preventing cell apoptosis. Thus, ludartin has therapeutic importance in spinal cord injury treatment.
\end{abstract}

\section{Introduction}

Spinal cord injury is characterised by the loss of function of limbs and body portion below the damaged region (Grigorean et al., 2009; Kwon et al., 2004). The cause may be attributed to mechanical stress as well as severe trauma (Grigorean et al., 2009; Kwon et al., 2004).

The primary injury is accompanied by secondary damage which includes the development of inflammation, onset of cell death by necrosis, apoptosis and autophagy (Liu et al., 1999). Therefore, secondary spinal cord injury is more harmful to the animals in comparison to the primary injury as it involves degeneration of the nervous system. The secondary spinal cord injury can be prevented as well as reversed by the use of chemotherapy and physiotherapy at an early stage (Sun et al., 2017).

However, at present the chemotherapeutic options for the management of secondary spinal cord injury are limited and thus the development of new molecules are of immense importance (Barinaga, 1996). Studies have demonstrated that neuronal cell apoptosis and necrosis play main role in tissue degeneration during secondary spinal cord injury (Barinaga, 1996). Glial and neuronal cell apoptosis have been observed as the major damaging processes which lead to secondary spinal cord injury in animal model and human tissue ( $\mathrm{Li}$ et al., 2000). Apoptosis and formation of cavities in the spinal cord of spinal cord injury patient has been found to get reduced by chemotherapy (Whalley et al., 2006).

The pathology of spinal cord injury is worsened by the activation of oxidative stress signalling pathway in animal model (Xiong et al., 2007). It is reported that NADPH oxidase expression in neuronal cells and microglia induces generation of reactive oxygen species (Bedard and Krause, 2007). The function of neurons 
under pathological conditions is modulated by the reactive oxygen species (Lo et al., 2007). Inhibition of NADPH oxidase or reactive oxygen species expression is one of the leading target for the treatment of neurological diseases.

Ludartin is a member of sesquiterpene lactone family and shows several pharmaceutical properties like antimicrobial, cytotoxic and antitumor (Rabe et al., 1985). Significant biological importance of ludartin has attracted its structure-activity relationship studies for the development of better and more active candidates. During these observations, the most active compound ludartin triazole was discovered (Rodriguez, 1976).

Over the past few decades, several strategies for the treatment and elucidation of molecular mechanism of spinal cord injury have been performed. However, there is no fully effective treatment till date available for spinal cord injury (Liu et al., 1999; Hausmann, 2003; Walker et al., 2012). One of the molecule, FK506 is known to possess neurotrophic and neuroprotective effect and has some importance in the functional recovery after spinal cord injury (Liu et al., 1999; Hausmann, 2003; Walker et al., 2012). Its neuroprotective effect is slow, thereby emphasis is laid on the development of a safe and efficient treatment for spinal cord injury. The current study investigates the effect of ludartin on expression of oxidative stress inducing factors, inflammatory cytokines and onset of apoptosis in spinal cord tissues of spinal cord injury rats.

\section{Materials and Methods}

\section{Chemicals}

Petroleum ether, ethyl acetate, chloroform and methanol were purchased from the (Merck, Germany). Dimethyl sulfoxide and $\mathrm{CDCl}_{3}$ were purchased from the Sigma-Aldrich (Merck KGaA, Germany).

\section{Plant material}

The aerial parts of the plant Stevia yaconensis var. subeglandulosa $(1.5 \mathrm{~kg}$ ) were collected in March, 2016 from the Tropical Botanical Garden, China. They were dried at room temperature and finely powdered. The plant material was authenticated by Zhe-Jun Zheng, a noted plant Taxonomist. The specimen sample was preserved in the Herbarium (voucher No. 106/16TBG).

\section{Method of extraction}

The powder (50 g) was extracted with chloroform at room temperature for 24 hours. The evaporation of chloroform afforded $2.5 \mathrm{~g}$ of the residue which was then subjected to partitioning between three solvents, methanol $(700 \mathrm{~mL})$, petroleum ether $(100 \mathrm{~mL})$ and distilled water $(200 \mathrm{~mL})$. The fraction of methanol-water was concentrated under reduced pressure and then treated with chloroform. After chloroform evaporation, $1 \mathrm{~g}$ residue was obtained which was then adsorbed on $1.5 \mathrm{~g}$ of silica gel. The extract was subjected to chromatographic separation using 60-120 mesh silica gel. The percentage yield of ludartin obtained was 1.98 .

The various fractions obtained from the column were pooled together, concentrated and then dried to obtain the white amorphous powder of the desired product. The fractions were subjected to thin layer chromatography.

\section{NMR data}

The product was characterised by ${ }^{1} \mathrm{H}$ NMR and ${ }^{13} \mathrm{C}$ NMR spectral techniques (Virginia et al., 1989). IRspectra on Perkin Elmer FT-IR spectrometer, NMR spectra on $400 \mathrm{MHz}$ Bruker spectrometers, mass analysis was carried out using Nexera UHPLC @ $130 \mathrm{Mpa}$ with SIL-30 AC Nexera-autosampler coupled to an LCMS 8030 tandem mass spectrometer manufactured by Shimadzu Corporation, Japan.

${ }^{1} \mathrm{H}$ NMR (400 MHz, $\left.\mathrm{CDCl}_{3}\right) \delta 6.124(1 \mathrm{H}, \mathrm{s}), 5.387(1 \mathrm{H}, \mathrm{s})$, $3.673(1 \mathrm{H}, \mathrm{t}, J=10.0 \mathrm{~Hz}), 3.396(1 \mathrm{H}, \mathrm{s}), 3.114(1 \mathrm{H}, \mathrm{d}, J=$ $10.8 \mathrm{~Hz}), 2.80(\mathrm{IH}, \mathrm{m}) 2.75$ ( 1H, d, J =17.5 Hz), $2.45(\mathrm{IH}$, $\mathrm{dq}, J=1.6, J=17.5 \mathrm{~Hz}), 2.1-2.3(2 \mathrm{H}, \mathrm{m}) 1.3-2.1(2 \mathrm{H}, \mathrm{m})$, $1.69(3 \mathrm{H}, \mathrm{s}), 1.67(3 \mathrm{H}, \mathrm{s}) ;{ }^{13} \mathrm{C} \mathrm{NMR}\left(400 \mathrm{MHz}, \mathrm{CDCl}_{3}\right) \delta$ 169.56, 139.43, 134.98, 133.83, 119.8 80.74, 67.17, 63.80, 54.53, 52.47, 33.96, 33.66, 25.83, 22.70, 19.06; IR cm-1: 1764, 1668, 1255, 825; EIMS: $269.00[\mathrm{M}+\mathrm{Na}]^{+}$.

The NMR was done at the State Key Laboratory of Magnetic Resonance and Atomic Molecular Physics, Wuhan Center for Magnetic Resonance, CAS Key Laboratory of Magnetic Resonance in Biological Systems, Wuhan Institute of Physics and Mathematics, Chinese Academy of Sciences, Wuhan, 430071 China.

\section{Establishment of spinal cord injury rat model}

Twenty-five male Sprague-Dawley rats (260-320 g) were supplied by the Experimental Animal Center belonging to the Guangxi Institute for Food and Drug Control. Immediately the rats were put into animal house with a controlled temperature of $22-24^{\circ} \mathrm{C}$ and humidity in the range $55 \pm 5 \%$. Rats were exposed to 12 hours light/dark cycle and provided free access to laboratory food and water. Rats were subjected to well set procedure involving spinal cord injury by compression in mid-thoracic region (Rivlin and Tator, 1978). Briefly, rats were anesthetized using 1\% urethane solution, placed on heating pad with temperature $37^{\circ} \mathrm{C}$ under sterile condition. Along the middle of the back, incision was made longitudinally to expose the muscles over the vertebral column. The dissection was carried out carefully in order to perform laminectomy on T6/ T7 vertebrae. The mid-thoracic region of spinal cord was covered with aneurysm clip for $45 \mathrm{sec}$ to keep the rat paraplegic. The incision was stitched and rat was kept on heat pad until gaining consciousness. 
Rats were divided into five groups of 5-each: control, spinal cord injury, $1 \mathrm{mg} / \mathrm{kg}, 2 \mathrm{mg} / \mathrm{kg}$ and $3 \mathrm{mg} / \mathrm{kg}$ treatment groups. The control group were not subjected to aneurysm clip surrounding but were given rest surgical procedure. Rats in the three treatment groups were administered $1 \mathrm{mg} / \mathrm{kg}, 2 \mathrm{mg} / \mathrm{kg}$ and $3 \mathrm{mg} / \mathrm{kg}$ doses of ludartin after spinal cord injury daily for 4 days intraperitoneally. Dimethyl sulfoxide was given to the rats of control and spinal cord injury groups in equal volume for 7 days after spinal cord injury through intraperitoneal route. On day 5, two rats from each group were sacrificed to extract spinal cord tissues for pathological examination. The rats were examined for behavioural analysis.

\section{Analysis of myeloperoxidase activity}

The activity of myeloperoxidase in the tissues of spinal cord was examined on day 5 using reported protocol (Hillegass et al., 1990). The rats anesthetized using 1\% urethane solution were subjected to extraction of spinal cord tissues which were subsequently treated with $\mathrm{K}_{2} \mathrm{PO}_{4}$ buffer $(50 \mathrm{mM})$ at $7.0 \mathrm{pH}$. The tissues were then centrifuged at $5,000 \times \mathrm{g}$ for $15 \mathrm{~min}$ at $4^{\circ} \mathrm{C}$ and the pellets obtained were put into $\mathrm{K}_{2} \mathrm{PO}_{4}$ buffer mixed with $\mathrm{C}_{16} \mathrm{H}_{33} \mathrm{~N}\left(\mathrm{CH}_{3}\right)_{3} \mathrm{Br}$. The pellets were subjected to freeze and thaw cycles under sonication followed by centrifugation for $5 \mathrm{~min}$ at $4^{\circ} \mathrm{C}$ at $5,000 \times \mathrm{g}$. Small volumes of the aliquots were reacted with $o$-dianisidine, $\mathrm{K}_{2} \mathrm{PO}_{4}$ buffer and hydrogen peroxide solution. Absorbance of the samples was recorded using spectrophotometer at $455 \mathrm{~nm}$ to determine myeloperoxidase activity in $\mathrm{U} / \mathrm{g}$.

\section{Determination of malondialdehyde and glutathione content}

The extracted tissue samples of spinal cord were treated with potassium chloride for homogenization. The level of malondialdehyde was then determined in the tissue homogenates by thiobarbituric acid treatment using the reported procedure (Beuge and Aust, 1978). Briefly, the homogenates were reacted with thiobarbituric acid and absorbance was measured at $535 \mathrm{~nm}$ wavelength. The activity of glutathione was analysed using the commercially available glutathione kit (Cayman, USA) in accordance with the manual protocol.

\section{Analysis of superoxide dismutase activity}

The activity of superoxide dismutase in the tissues of spinal cord was determined using spectrophotometer (UV-2100; Shimadzu Co. Ltd., China). The supernatant was treated with xanthine in accordance with known procedure (Oyanagui, 1984). Measurement of absorbance was performed at $557 \mathrm{~nm}$ wavelength using a spectrophotometer (Rabiei and Rafieian, 2014).

\section{Histopathological analysis}

On day 5, rats were sacrificed after $1 \%$ urethane anesthetization to perfuse paraformaldehyde (4\%).
From the spinal cord, $2 \mathrm{~mm}$ segments were extracted at caudal and rostral site to the injury. The tissue sections were placed for 20 hours in paraformal-dehyde $(4 \%)$ and subsequently transferred to sucrose solution. The tissues were then sliced into $20 \mu \mathrm{m}$ sections axially and subjected to permeabilization in for $45 \mathrm{~min}$ in Triton $X-100$. The sections were then incubated for 2 hours at $37^{\circ} \mathrm{C}$ in donkey serum. Again the sections were incubated for overnight at $4^{\circ} \mathrm{C}$ with antibodies against NeuN (dilution 1:100; catalog number ab104224; Abcam, UK) and TUNEL. Then the incubation was carried out at room temperature for 1 hour with secondary antibodies (Alexa Fluor 488 donkey anti-mouse IgG; diluted 1:100; ab150105; Abcam).

\section{Western blot analysis}

The spinal cord tissues of the rats after homogenization were centrifuged to obtain the tissue lysates. The protein content in the lysates was determined by Bradford method employing the commercially available BCA protein assay kit (Sigma, USA). Separation of the proteins was performed on $10 \%$ sodium dodecyl sulfate polyacrylamide gel by electrophoresis (SDS-PAGE). The proteins were transferred onto the nitro-cellulose membrane which were blocked by treatment for 2 hours with $5 \%$ non-fat milk at room temperature. The membranes were then incubated for overnight with primary antibodies at a temperature of $4^{\circ} \mathrm{C}$. The primary antibodies used were against: Bax (catalog number ab32503; dilution 1:1,000), Bcl-2 (catalog number ab32124; dilution 1:500), tumor necrosis factor-a (catalog number ab6671; dilution 1:500), interleukin-1 $\beta$ (catalog ab2105; dilution 1:500), nterleukin-6 (catalog number ab6672; dilution 1:500) and $\beta$-actin (catalog number ab8227; dilution 1:1,000) all from Abcam. After incubation membranes were washed and then subjected to incubation for 1 hour at $37^{\circ} \mathrm{C}$ with goat anti-rabbit IgG secondary antibodies (catalog number ab6721; Abcam). The band illumination was carried out using ECL system (Santa Cruz Biotechnology, USA).

\section{Analysis of rat behaviour on inclined plane and motor function}

The behaviour of rats on day 2, 4, 6, 8, 10, 12 and 14 after spinal cord injury was analysed by examining their ability to balance on wooden beams kept in inclined position. The protocol used was based on the method with slight modification (Rivlin and Tator, 1977). The device consisted of a plate which could be adjusted through an angle of $0-90^{\circ}$. The recovery of motor function of the rat limbs was analysed using Basso, Beattie and Bresnahan scale. The scale consisted of 21 divisions for assigning motor limb recovery score (Basso et al., 1995). On this scale the normal rats were assigned 21 and those with paralysed limbs a score of 0 . The method involved allowing the rats to move forcibly in open field for $30 \mathrm{~min}$ on day 2, 4, 6, 8, 10, 12 and 14 
after spinal cord injury and examine their hind limb movements. The hind limb score determined was expressed as the average of three measurements.

\section{Statistical analysis}

The values presented are the mean of SD of three independently carried out experiments. The statistical differences among the groups were analysed by one-way ANOVA followed by Bonferroni correction. Statistically significant differences were taken at the values of $\mathrm{p}<0.05$.

\section{Results}

\section{Expression of oxidative stress factors}

Analysis of myeloperoxidase in the rats of spinal cord injury showed significantly $(p<0.05)$ higher level in comparison to the rats of control group (Figure 1). However, ludartin treatment of spinal cord injury rats decreased the expression of myeloperoxidase in dosage based manner in tissues of spinal cord. The level of myeloperoxidase in the rats of $1 \mathrm{mg} / \mathrm{kg}$ group was close to those of untreated group and those of $3 \mathrm{mg} / \mathrm{kg}$ group almost equal to the control rats. The level of myeloperoxidase in rats of $2 \mathrm{mg} / \mathrm{kg}$ group was in between those of $1 \mathrm{mg} / \mathrm{kg}$ and $3 \mathrm{mg} / \mathrm{kg}$ groups. The expression of malondialdehyde in spinal cord injury rats was also found to be significantly $(p<0.05)$ higher than those of control rats. In the spinal cord injury rats treatment with ludartin inhibited expression of malondialdehyde in concentration based manner. Treatment with $3 \mathrm{mg} / \mathrm{kg}$ dose of ludartin significantly inhibited spinal cord injury induced increase of malondialdehyde in the rats. The expression of glutathione and superoxide dismutase was found to be significantly reduced in the rats of untreated spinal cord injury group $(\mathrm{p}<0.02)$. Ludartin treatment of spinal cord injury rats significantly prevented decrease of glutathione and superoxide dismutase. There was no significant difference in glutathione and superoxide dismutase expression in the rats of control and $3 \mathrm{mg} / \mathrm{kg}$ ludartin treatment groups.

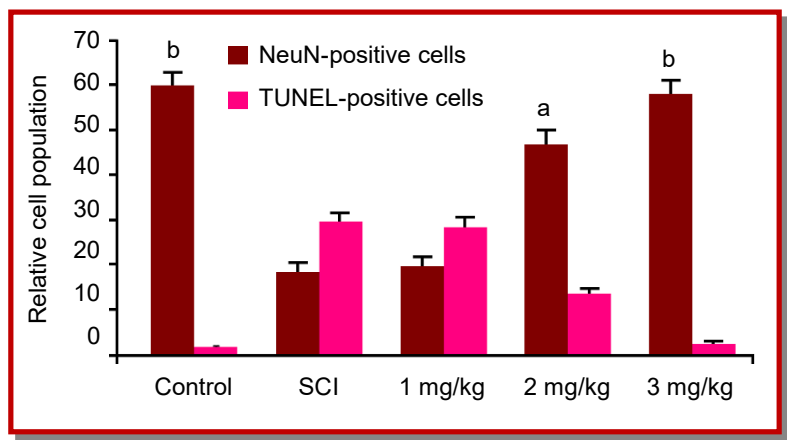

Figure 2: Regulatory effect of ludartin on NeuN and TUNELpositive cell population in spinal cord injury (SCI) rats. The tissues were examined after immunofluorescent staining and examined for NeuN and TUNEL-positive cell population. The expressed values are average of three results carried out independently. ${ }^{a} \mathrm{p}<0.05$ and ${ }^{b} \mathrm{p}<0.02$ vs. untreated spinal cord injury rats

\section{NeuN-positive and TUNEL-positive cells}

In spinal cord injury rats, the level of NeuN-positive cells was found to be markedly lower than those of control rats. However, ludartin treatment of spinal cord injury rats exhibited inhibitory effect on reduction of NeuN-positive cells. There was no significant difference between control rats and $3 \mathrm{mg} / \mathrm{kg}$ ludartin treatment group in the level of NeuN-positive cells (Figure 2). Examination of TUNEL-positive cells led to the identification of higher proportion of TUNEL-positive cells in spinal cord injury rat than those in control group. Treatment of spinal cord injury rats with ludartin prevented spinal cord injury-mediated increase of TUNELpositive cell population.

\section{Expression of caspase-3, Bax and $\mathrm{Bcl}-2$}

Analysis of caspase-3 and Bax level showed marked enhancement in the rats of spinal cord injury group than those of control rats. In the spinal cord injury rat treatment with ludartin prevented enhancement of caspase-3 and Bax content in comparison to the untreated rats (Figure 3). The expression level of Bcl-2 was found to be suppressed in the tissues of spinal cord
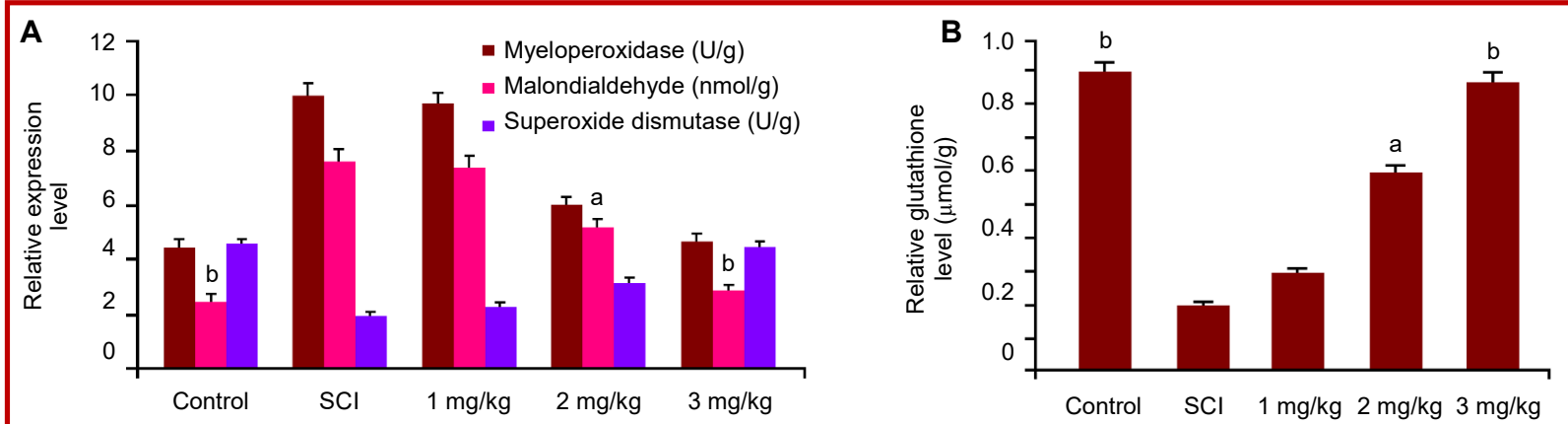

Figure 1: Changes in oxidative factor expression (myeloperoxidase, malondialdehyde and superoxide dismutase) (A) and glutathione (B) levels in rat with spinal cord injury (SCI) by ludartin. The values represented are the mean obtained from three independently performed experiments. ${ }^{a} \mathrm{p}<0.05$ and ${ }^{b} \mathrm{p}<0.02$ vs. untreated spinal cord injury rats 


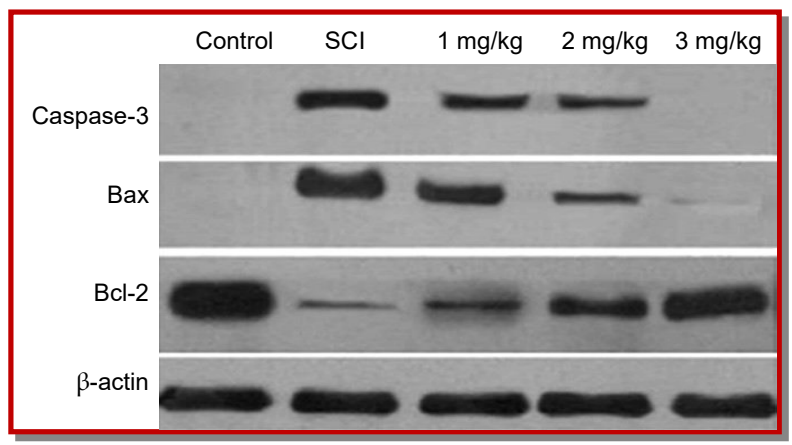

Figure 3: Regulation of caspase-3, Bax and Bcl-2 level by ludartin in spinal cord injury rats. Relative expression of caspase-3, Bax and Bcl-2 in the rat tissues was analysed using Western blotting

injury rats. However, ludartin treatment caused increase in the level of Bcl-2 in spinal cord injury rats than those of untreated group (Figure 3).

Tumor necrosis factor-a, interleukin-1 $\beta$ and interleukin-6 expression

The tissues of spinal cord injury rats showed marked enhancement of tumor necrosis factor- $\alpha$, interleukin- $1 \beta$ and I interleukin-6 level than the rats in control group. Among the three ludartin treatment groups, the level of these three cytokines was reduced comparably equal to that of control rats in $3 \mathrm{mg} / \mathrm{kg}$ treatment rats (Figure 4). Thus, ludartin treatment reversed the effect of spinal cord injury on tumor necrosis factor- $\alpha$, interleukin- $1 \beta$ and interleukin- 6 level in the tissues of rats in dosage

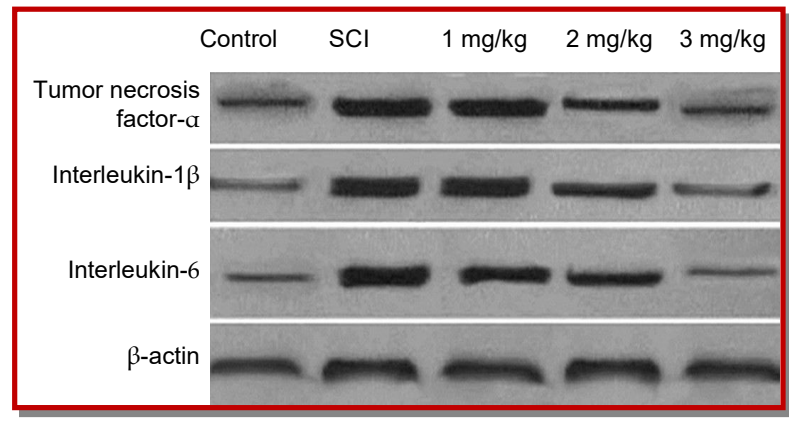

Figure 4: Effect of ludartin on cytokines. Use of Western blot assay was made for analysis of tumor necrosis factor- $\alpha$, interleukin- $1 \beta$ and interleukin-6 level in rat tissues. The TNF stands for tumor necrosis factor and IL stands for interleukin

based manner.

\section{Motor function of spinal cord}

Difference in the movement of rats was examined using plane inclination method on day 2, 4, 6, 8, 10, 12 and 14 after spinal cord injury. Rats in untreated spinal cord injury group showed very low plane inclination and Basso, Beattie and Bresnahan locomotion score than rats of control group. Rats in the ludartin $(3 \mathrm{mg} / \mathrm{kg})$ treatment group showed plane inclination and Basso, Beattie and Bresnahan locomotion score almost equal to the rats in control group (Figure 5). This ludartin treatment improved the motor function of spinal cord in the spinal cord injury rats.

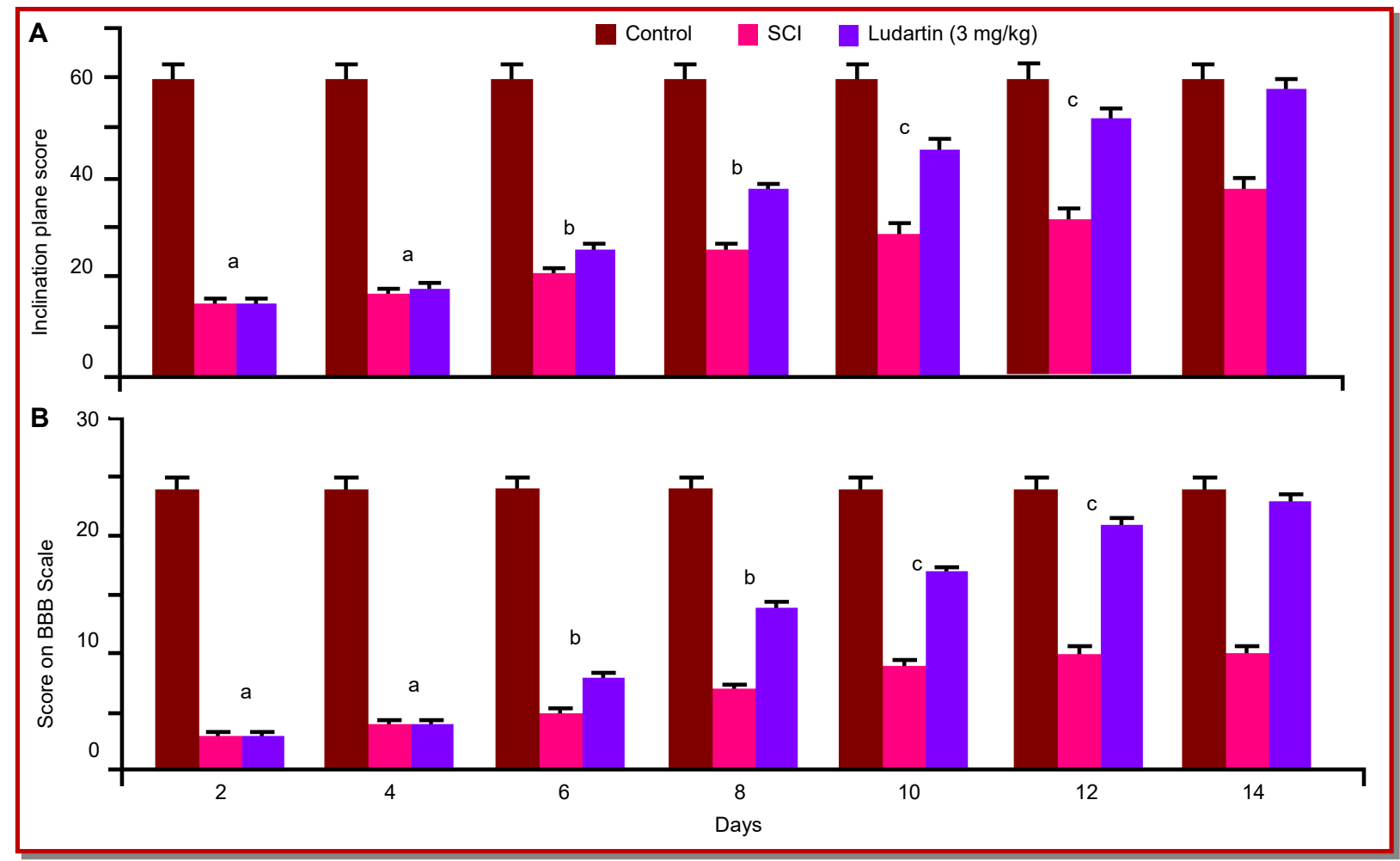

Figure 5: Motor function in spinal cord injury (SCI) rats by ludartin. (A) Score on inclination plane on day 2, 4, 6, 8, 10, 12 and 14 after spinal cord injury. (B) Score on Basso, Beattie and Bresnahan scale on day 2, 4, 6, 8, 10, 12 and 14 after spinal cord injury. Presented values are average of three experiments. ${ }^{a} p<0.05,{ }^{b} p<0.02$ and $c p<0.01$ vs. untreated spinal cord injury rats 


\section{Discussion}

In the current study, investigation of therapeutic role of ludartin on spinal cord injury in rat model was planned. It was observed that ludartin prevented neuronal damage by inhibiting expression of inflammatory cytokines and pro-apoptotic factors. Ludartin treatment considerably improved locomotion of spinal cord injury rats which was clear by high inclination plane and Basso, Beattie and Bresnahan scores.

Spinal cord injury leads to neuronal apoptosis and subsequent degradation of neurological system (Blight, 1991). Study of the mechanism of primary and secondary spinal cord injury has revealed the involvement of several cellular signalling pathways (Song et al., 2013). The major contributing pathway which causes neuronal damage and development of secondary spinal cord injury has been identified as oxidative stress (Juurlink and Paterson, 1998). The neutrophils and monocytes contain a positively charged protein called myeloperoxidase which catalyses oxidative damage to nerve cells (Juurlink and Paterson, 1998). Another oxidative stress inducing factor is malondialdehyde which is generated as the final product of lipid peroxidation (Juurlink and Paterson, 1998). However, the two enzymes which act as scavengers for free radicals present in cells are glutathione and superoxide dismutase (Juurlink and Paterson, 1998). Studies have demonstrated that inhibition of oxidative stress can prevent the neuronal cell damage and development of secondary injury (Impellizzeri et al., 2011). In the current study, level of myeloperoxidase and malondialdehyde was found to be enhanced and the activation of glutathione and superoxide dismutase was suppressed in spinal cord injury rats. However, ludartin treatment prevented spinal cord injury-mediated enhancement of myeloperoxidase and malondialdehyde as well suppression of glutathione and superoxide dismutase activity. These findings indicated that ludartin exhibits its effect inhibiting oxidative damage to neuronal cells.

Ludartin, a member of guaianolides has been isolated as a mixture along with 11,13-dihydroderivative from Artemisia caruthii and in pure form from Stevia yaconensis var. subeglandulosa and from Artemisia filatovae.

Onset of secondary spinal cord injury has been shown cause production of apoptosis inducing factors and inflammatory cytokines which are the activators of MAPK pathways (Song et al., 2013). The process of inflammation at the site of injury involves higher expression of tumor necrosis factors- $\alpha$, interleukin- $1 \beta$ and interleukin-6 (Fehlings and Nguyen, 2010). It is well known that the level of inflammatory cytokines increases significantly in animals after spinal cord injury (Impellizzeri et al., 2012). Inhibition of inflammatory factor expression is considered to be of great importance for the treatment of spinal cord injury. In the current study the level of tumor necrosis factors- $\alpha$, interleukin-1 $\beta$ and interleukin-6 was found to be markedly higher in the spinal cord injury rats. However, the level of these three cytokines was decreased and maintained equal to that in control rats by treatment with ludartin.

During spinal cord injury the neuronal damage is predominantly associated with the induction of apoptosis (Rong et al., 2012). The process of cell apoptosis involves various factors among which caspase 3 and Bax are the important components (Wei et al., 2012). In the present study level of caspase- 3 and Bax in tissues of spinal cord of spinal cord injury rats were found to be markedly higher which suggests that neuronal degradation involves apoptosis. Ludartin treatment, however, significantly prevented increase of caspase- 3 and Bax levels in spinal cord injury rats. The level of Bcl-2 was increased by treatment of spinal cord injury rats with ludartin. Treatment of spinal cord injury rat with ludartin improved locomotion by increasing inclination plane and Basso, Beattie and Bresnahan scores.

\section{Conclusion}

Ludartin treatment prevents neuronal damage and improves locomotion in spinal cord injury rats by inhibiting inflammatory factor, cytokine and apoptosis onset. Thus, ludartin has therapeutic importance and can be used for spinal cord injury treatment.

\section{Ethical Issue}

To perform experiments using animal, the approval was obtained from the Animal Care and Use Committee of Xi'an Medical University.

\section{Conflict of Interest}

The authors declare no conflict of interest in the publication of these results.

\section{Acknowledgement}

The support from the Head, Department of Orthopedics, Wuwei People's Hospital, China is acknowledged.

\section{References}

Barinaga M. New view of spinal cord injury. Science 1996; 274: 1466.

Bedard K, Krause KH. The NOX family of ROS-generating NADPH oxidases: Physiology and pathophysiology. Physiol Rev. 2007; 87: 245-313.

Basso DM, Beattie MS, Bresnahan JC. A sensitive and reliable 
locomotor rating scale for open field testing in rats. J Neurotrauma 1995; 12: 1-21.

Blight AR. Morphometric analysis of blood vessels in chronic experimental spinal cord injury: Hypervascularity and recovery of function. J Neurol Sci 1991; 2: 158-74.

Beuge JA, Aust SD. Microsomal lipid peroxidation. Methods Enzymol. 1978; 52: 302-10.

Fehlings MG, Nguyen DH. Immunoglobulin G: A potential treatment to attenuate neuroinflammation following spinal cord injury. J Clin Immunol. 2010; 30: S109-12.

Grigorean VT, Sandu AM, Popescu M, Iacobini MA, Stoian R, Neascu C, Strambu V, Popa F. Cardiac dysfunctions following spinal cord injury. J Med Life. 2009; 2: 133-45.

Hausmann ON. Post-traumatic inflammation following spinal cord injury. Spinal Cord. 2003; 41: 369-78.

Hillegass LM, Griswold DE, Brickson B, Albrightson-Winslow C. Assessment of myeloperoxidase activity in whole rat kidney. J Pharmacol Methods. 1990; 24: 285-95.

Impellizzeri D, Mazzon E, Esposito E, Paterniti I, Bramanti P, Cuzzocrea S. Effect of apocynin, an inhibitor of NADPH oxidase, in the inflammatory process induced by an experimental model of spinal cord injury. Free Radic Res. 2011; 45: 221-36.

Impellizzeri D, Esposito E, Mazzon E, Paterniti I, Di Paola R, Bramanti P, Morittu VM, Procopio A, Perri E, Britti D, Cuzzocrea S. The effects of a polyphenol present in olive oil, oleuropein aglycone, in an experimental model of spinal cord injury in mice. Biochem Pharmacol. 2012; 83: 1413-26.

Juurlink BH, Paterson PG. Review of oxidative stress in brain and spinal cord injury: Suggestions for pharmacological and nutritional management strategies. J Spinal Cord Med. 1998; 21: $309-34$

Kwon BK, Tetzlaff W, Grauer JN, Beiner J,d Vaccaro AR. Pathophysiology and pharmacologic treatment of acute spinal cord injury. Spine J. 2004; 4: 451-64.

Liu D, Xu GY, Pan E, McAdoo DJ. Neurotoxicity of glutamate at the concentration released upon spinal cord injury. Neuroscience 1999; 93: 1383-89.

Li GL, Farooque M, Olsson Y. Changes of Fas and Fas ligand immunoreactivity after compression trauma to rat spinal cord. Acta Neuropathol. 2000; 100: 75-81.

Lo W, Bravo T, Jadhav V, Titova E, Zhang JH, Tang J. NADPH oxidase inhibition improves neurological outcomes in surgically-induced brain injury. Neurosci Lett. 2007; 414: 228-32.

Oyanagui Y. Revaluation of assay methods and establishment of kit for superoxide dismutase activity. Anal Biochem.
1984; 142: 290-96

Rabiei Z, Rafieian M. Effects of Zizyphus jujuba extract on motor coordination impairment induced by bilateral electric lesions of the nucleus basalis of meynert in rat. Physiol Pharmacol. 2014; 17: 469-77.

Rabe J, Hoffmann HMR. Synthesis and biological activity of amethylene- $\gamma$-butyrolactones. Angew Chem Int Ed Engl. 1985; 24: 94

Rivlin A, Tator $\mathrm{CH}$. Effect of duration of acute spinal cord compression in a new acute injury model in the rat. Surg Neurol. 1978; 10: 38-43.

Rivlin AS, Tator $\mathrm{CH}$. Objective clinical assessment of motor function after experimental spinal cord injury in the rat. J Neurosurg. 1977; 47: 577-81.

Rong W, Wang J, Liu X, Jiang L, Wei F, Hu X, Han X, Liu Z. Naringin treatment improves functional recovery by increasing BDNF and VEGF expression, inhibiting neuronal apoptosis after spinal cord injury. Neurochem Res. 2012; 37: 1615-23.

Rodriguez E. Towers GHN, Mitchell JC. Biological activities of sesquiterpene lactones. Phytochemistry 1976; 15: 1573.

Song Y, Liu J, Zhang F, Zhang J, Shi T, Zeng Z. Antioxidant effect of quercetin against acute spinal cord injury in rats and its correlation with the p38MAPK/iNOS signaling pathway. Life Sci. 2013; 92: 1215-21.

Sun Y, Gong F, Yin J, Wang X, Wang X, Sun Q, Zhu Z, Su X, Zheng J, Liu L, Li Y, Hu X, Li J. Therapeutic effect of apocynin through antioxidant activity and suppression of apoptosis and inflammation after spinal cord injury. Expt Therapeutic Med. 2017; 13: 952-60.

Virginia EA, Juan CO, Roberto RG, Edmundo AR, Virgil LG, Alicia BG, Werner H. 10-Epideoxycumambrin B and other constituents of Stevia Yaconensis var. Subeglandulosa. Phytochemistry 1989; 28: 1925-29.

Walker CL, Walker MJ, Liu NK, Risberg EC, Gao X, Chen J, Xu XM. Systemic bisperoxovanadium activates Akt/mTOR, reduces autophagy and enhances recovery following cervical spinal cord injury. PLoS One. 2012; 7: e30012.

Wei H, Teng H, Huan W, Zhang S, Fu H, Chen F, Wang J, Wu C, Zhao J. An up-regulation of SENP3 after spinal cord injury: Implications for neuronal apoptosis. Neurochem Res. 2012; 37: 2758-66.

Whalley K, O'Neill P, Ferretti P. Changes in response to spinal cord injury with development: vascularization, hemorrhage and apoptosis. Neuroscience 2006; 137: 821-32.

Xiong Y, Rabchevsky AG, Hall ED. Role of peroxynitrite in secondary oxidative damage after spinal cord injury. J Neurochem. 2007; 100: 639-49.

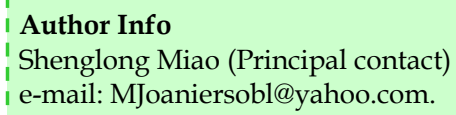

